\title{
Next Steps in Space Travel and Colonization: Terraforming, Ectogenesis, Nano Spacecraft and Avatars
}

\author{
Martin Braddock* \\ Mansfield and Sutton Astronomical Society, UK \\ *Corresponding author: Martin Braddock, Sherwood Observatory, Mansfield and Sutton Astronomical Society, Coxmoor Road, Sutton-in-Ashfield, \\ Nottinghamshire, UK
}

Submission: August 27, 2018; Published: August 31, 2018

\begin{abstract}
The routine detection of exoplanets has started to yield a candidate list of new worlds potentially capable of harboring or sustaining life. To date 3775 exoplanets have been confirmed contained within 625 planetary systems. Of the 3775 planets, 55 are deemed suitable to support life of which 1 is subterran (0.1-0.5 ME or 0.4-0.8RE; ME=Earth mass, and RE=Earth radius), 22 are terran (0.5-5ME or 0.8-1.5RE) and 32 are superterran (5$10 \mathrm{ME}$ or 1.5-2.5RE) [1,2]. Facilitated in part to date by the Gaia [3,4] and Kepler space telescopes [5,6], the launch of the Transiting Exoplanet Survey Satellite (TESS) on April $18^{\text {th }}, 2018[7,8]$ which will monitor more than 200,000 stars for temporary drops in brightness caused by planetary transits further increases what is surely a highly likely outcome; identification of a set of candidates for further exploration. In 2018, the principle driver for identification of an 'Earth 2.0', a planet very close to Earth characteristics is scientific curiosity and to determine whether life exists in, or beyond our Solar system. However, there is now growing evidence that the activities of humans as of 12,000yrs ago and to the present day have increased extinction rates of many species and that we are experiencing the $6^{\text {th }}$ extinction level event (ELE) or the Holocene extinction [9,10]. The consequences of this latest ELE may add urgency to considering other planets for where mankind can migrate and settle, in part as a potential staging post for further exploration and in part as a failsafe should Earth become inhospitable to supporting life as we know it today. For both near (solar system) and deep (beyond solar system) space travel, the ergonomic challenges facing manned spaceflight for both human physiological and psychological adaptation to microgravity are well understood and countermeasures for and mitigation of the effects of microgravity are being developed [11], which include generation of artificial or simulated gravity in space [12-14]. The longevity of human lifespan is an as yet unsurmountable obstacle for reaching even the nearest stars with propulsion technologies available today and strategies are being considered which may prolong functional lifespan [15]. An alternative route to exploring deep space is to deploy unmanned space probes which combine the evolving fields of artificial intelligence, design and deployment of nano spacecraft and the futuristic concept of sending humans as avatars on small lightweight spacecraft as an e-crew. Taken together, it is possible to construct two parallel lines of thinking and simple decision matrices which utilize manned or unmanned space craft and in the first review we will consider their application to both near and far distance space travel missions.
\end{abstract}

\section{Introduction}

Picture this scenario. Climate change, accentuated by human activity has and will irrevocably alter the world we live in and today, we are undergoing the Earth's $6^{\text {th }}$ extinction level event (ELE)the Holocene event and studies have shown that the current rate of extinction of species is estimated at 100 to 1,000 times higher than natural background rates over the last $10,000 \mathrm{yr}[9,10]$. The inhabitable land mass of the Earth will decrease as sea levels rise and islands in the Pacific Ocean have disappeared [16]. Dramatic weather extremes have led to crop failure and deforestation has destabilized soil structure in large swathes of arable land which have been washed away by heavy rain. Widespread food and water shortages have triggered global conflict and the fabric and integrity of human civilization built over the last $5,000 \mathrm{yr}$ is under threat of disintegration. Clearly this is, hopefully, an overly negative scenario and mitigation measures are in place around the globe to minimize the consequences of human activities on climate change [17-19]. ELEs may also be caused by events that are out with our control such as an asteroid strike or atmospheric alteration by release of methane via a clathrate gun, eruption of a super-volcano or depletion of selenium. The is evidence, for example linking volcanic activity, selenium depletion, and asteroid strike and geomagnetic reversals with previous extinction level events [20,21] and taken together as our technology develops, it would seem prudent for our species to plan for the ultimate species migration; either to a nearby or distant planet.

\section{Proposed events enabling colonization, near and deep space travel}

Fourteen events or activities have been considered which may influence if not drive our ability to reach and colonise other worlds (Table 1) and some of these have been presented in a 
previous publication [15]. The likelihood and potential impact of these events will be discussed within this review which will permit a comparison and a useful summary as a basis for further research. I will attempt to set this in the context of what I believe to be achievable today (Table 1a) and over the next 100yr (Table 1b) and draw where possible on substantiating evidence from the scientific literature, taking licence where necessary to speculate on the nature of future scientific developments.

Table 1a: Proposed events enabling space travel and colonization for an arbitrary date in 2018.

\begin{tabular}{|c|c|c|c|c|c|c|}
\hline $\mathbf{A}$ & \multicolumn{6}{|c|}{ Impact } \\
\hline \multirow{7}{*}{ Likelihood } & & Negligible & Minor & Moderate & Significant & Major \\
\hline & Very likely & - & & Media hype or falsehood & Exoplanet candidate ID & - \\
\hline & Likely & - & Travel to Mars & AG generation in space & - & - \\
\hline & Possible & - & & Nano spacecraft deployment & Colonisation of Mars & - \\
\hline & Unlikely & - & Ectogenesis & Hibernation & Negligible senescence & - \\
\hline & \multirow{2}{*}{ Very unlikely } & - & Terraforming & \multirow{2}{*}{-} & Cryogenics & \multirow{2}{*}{$\begin{array}{l}\text { Manned Interstellar } \\
\text { (FTL) travel }\end{array}$} \\
\hline & & - & Mind uploading & & Worldships & \\
\hline
\end{tabular}

Table 1b: Proposed events enabling space travel and colonization for an arbitrary date in 2118.

\begin{tabular}{|c|c|c|c|c|c|c|}
\hline B & \multicolumn{6}{|c|}{ Impact } \\
\hline \multirow{8}{*}{ Likelihood } & & Negligible & Minor & Moderate & Significant & Major \\
\hline & \multirow[t]{2}{*}{ Very likely } & & & \multirow[t]{2}{*}{ Travel to Mars } & AG generation in space & \multirow[t]{2}{*}{ Exoplanet candidate ID } \\
\hline & & & & & Colonisation of Mars & \\
\hline & Likely & & & Hibernation & Mind uploading & $\begin{array}{c}\text { Nano spacecraft deployment } \\
\text { and data retrieval }\end{array}$ \\
\hline & Possible & & $\begin{array}{l}\text { Media hype or } \\
\text { falsehood }\end{array}$ & Ectogenesis & Negligible senescence & \\
\hline & Unlikely & & & & & Terraforming \\
\hline & \multirow{2}{*}{ Very unlikely } & & & & Worldships & \multirow{2}{*}{$\begin{array}{c}\text { Manned Interstellar (FTL) } \\
\text { travel }\end{array}$} \\
\hline & & & & & Cryogenics & \\
\hline
\end{tabular}

\section{Parallel thought processes for design options enabling near and deep space travel and colonization}

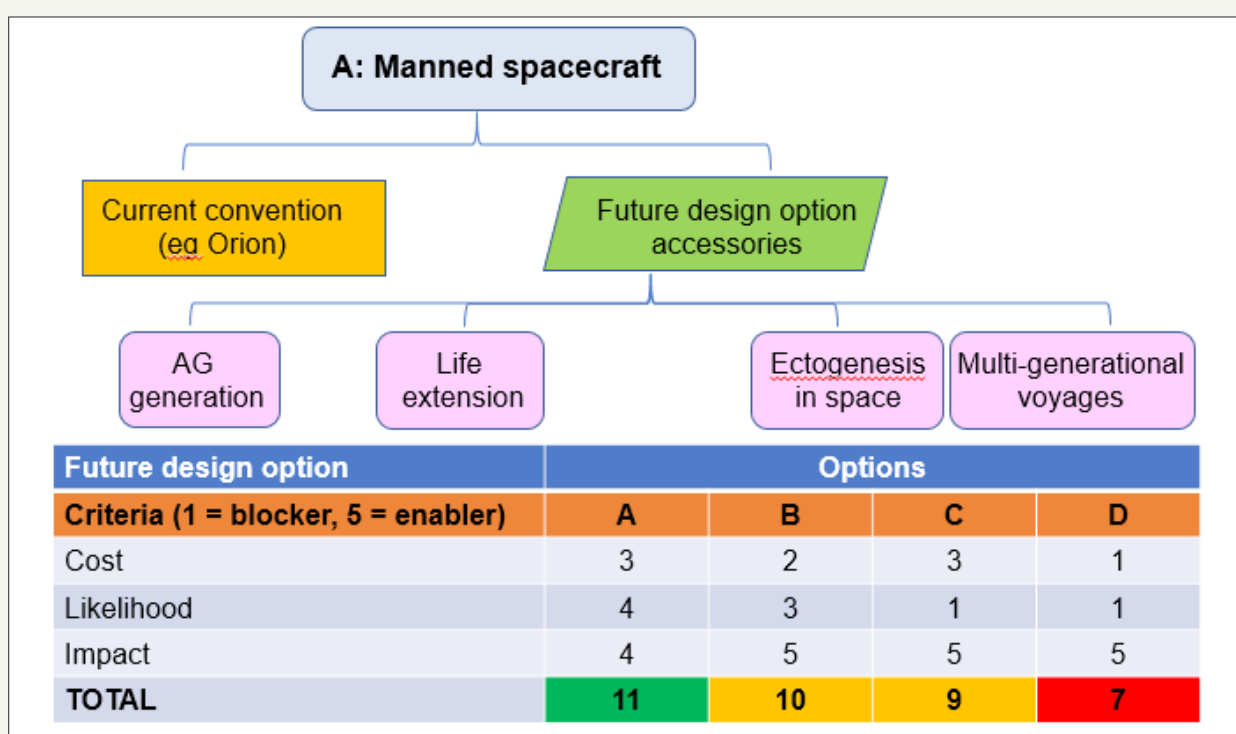

Figure 1A: Future design option accessories and decision matrices for manned spacecraft. 
Figure 1 considers potential option accessories for both manned and unmanned spacecraft and a simple decision matrix for each process. Although options are considered separately for manned spacecraft travel (Figure 1A), it is likely that several options would be deployed, for example space based ectogenesis and other routes to extend life summarised previously [15] may need to be complemented by an environment of artificial gravity (AG). Out of the accessorized options presented, deployment of AG to act as a countermeasure to the effects of microgravity appears the most favourable option and this is consistent with the recent announcement of an International Roadmap for Artificial Gravity
Research [13] which describes the ambition for multiple space agencies to safely develop and adapt a spacecraft to provide a habitat in which astronauts will experience AG.

For unmanned spacecraft (Figure 1B), two options are considered, both involve nano spacecraft which are discussed in more detail below and the first option is without and the second option is with a higher form of artificial intelligence (AI) or avatar. With our present level of technology, a nano spacecraft lacking an AI payload is more favorable, this is expected to change over the next $100 \mathrm{yr}$ and is also further discussed below.

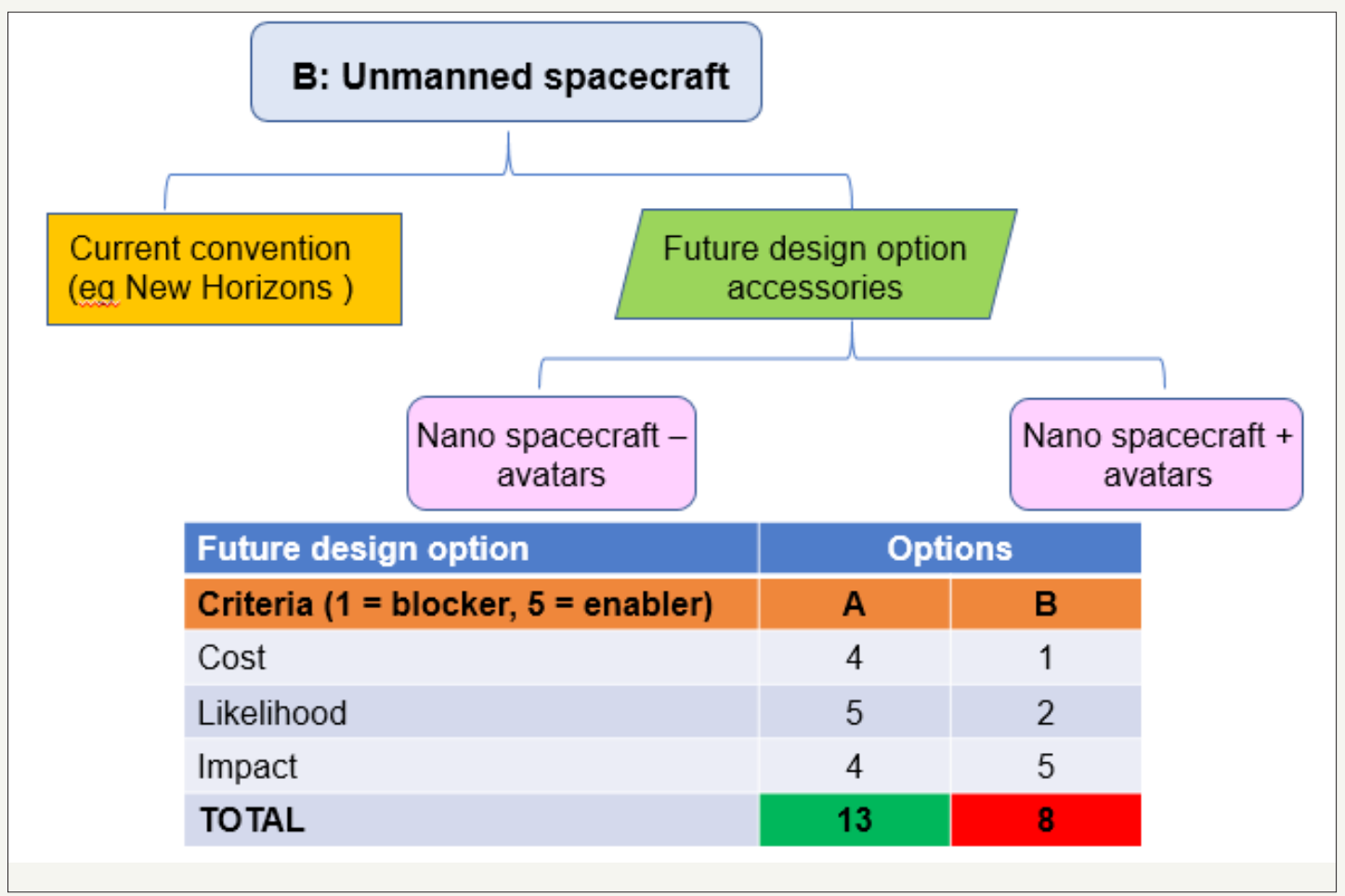

Figure 1B: Future design option accessories and decision matrices for unmanned spacecraft.

\section{Terraforming the solar system}

Terraforming is the process whereby the atmospheric composition, temperature, pressure and surface topology of a planet, moon, or other body is deliberately modified to be similar to that of Earth such that it may be habitable by Earth-like life. This is a very speculative, but possibly valid solution for a nearby alternative for human habitation. Table 2a summarises the principle pros and cons of terraformation. Although Venus, Titan and other Saturnian moons and the moons of Jupiter have been proposed as possible candidates for terraforming, Mars has traditionally been regarded as the prime candidate [22] and Table $2 \mathrm{~b}$ presents the principle pros and cons which need to be evaluated when considering Mars as a target planet. The primary aim in terraforming Mars is to increase the temperature to that where liquid water may exist, and this may be accomplished by the introduction of greenhouse gases such as carbon dioxide. However, although there are clear advantages in considering Mars, due in part to its proximity to Earth, a very recent study has suggested there is only enough carbon dioxide locked within the planet which when released would produce an atmosphere of $\sim 7 \%$ that of Earth which would not provide enough gas to produce atmospheric warming [23]. It appears, at least with today's technology, that Mars is no longer the prime terraforming candidate in the Solar system and this may re-stimulate interests in Venus, built in part upon a better understanding of planetary rotation speed and the possibility of being able to support life in the distant past [24].

Opinion: Travel to Mars is considered likely to happen today and very likely within the next $100 \mathrm{yr}$. Terraforming Mars is a very unlikely activity to commence today and based upon current evidence will remain an unlikely activity over the next $100 \mathrm{yr}$. The consequence is that this has a severe impact on the chances of colonization of another planet, as although the process to establish a platform for photosynthesis is likely to take up to $100,000 \mathrm{yr}$, the lack of a suitable target planet renders this option unfavourable, even at the stage of planning. 


\section{Manned spacecraft and the potential for crew generation by controlled ectogenesis}

Ectogenesis is the growth process of an organism in an artificial environment outside the body in which it would normally be found, such as the growth of an embryo or fetus outside the mother's body. It requires the development of an artificial uterus and placenta and it could be envisaged that the process for producing a crew by in vitro fertilisation (IVF) and implantation into an artificial uterus could be conceived and developed within 20-30yr of arrival at a target exoplanet by automation. Since the first successful birth of a child after IVF treatment in 1978, in 2012 it was estimated that five million and in July 20188 million children had been born worldwide using IVF and other assisted reproduction techniques $[25,26]$, illustrating this technique to be standard medical practice. The potential for human beings born prematurely and to be kept alive outside of the womb has received a lot of attention over the

Table 2a: Terraforming the Solar system. last decade, with the primary intent to allow very premature babies to develop normally and to lead a life to the same quality as babies born at full term [27]. The minimum survivable human gestational age is about 22 weeks and to date, our limited understanding of placental function which enables development and full functional capacity of the lungs is supported by the high associated rates of chronic lung disease and other complications of organ immaturity, particularly in infants born before 28 weeks. Recently, the development of an extra-uterine system has been reported. The system incorporates a pumpless oxygenator circuit connected to the foetus of a lamb via an umbilical cord maintained within a closed 'amniotic fluid' circuit that closely reproduces the environment of the uterus. Foetal lambs developmentally equivalent to the extreme premature human infant were supported in this system for up to 4 weeks where they maintained normal circulatory parameters and demonstrated normal somatic growth, lung maturation, brain growth and myelination [28].

\begin{tabular}{|c|c|c|}
\hline A & Pros & Cons \\
\hline & A unifying project for Earth's people & Enormous time scales (up to $100,000 \mathrm{yr}$ for photosynthesis) \\
\hline & $\begin{array}{l}\text { Would provide a survival colony in the face of an Earth disaster and/or a stage } \\
\text { post for further travel }\end{array}$ & Human physiology will evolve differently \\
\hline & Ecosynthesis allows free movement with full face masks & $\begin{array}{l}\text { Legal and administrative challenges } \\
\text { •Who lives there and how is it decided? } \\
\qquad \text { Who owns it? } \\
\text { •Who controls it-Earth or autonomous? }\end{array}$ \\
\hline & Stimulate technological developments eg colonists could live of local biomass & $\begin{array}{l}\text { Less attention to important terrestrial problems eg climate } \\
\text { change }\end{array}$ \\
\hline & Better use of resources than building military stockpiles & \multirow{2}{*}{ Very long-term economic payback } \\
\hline & Travel distance and time not limited by human lifespan & \\
\hline
\end{tabular}

Table 2b: Terraforming Mars.

\begin{tabular}{|c|c|c|}
\hline B & Pros & Cons \\
\hline \multirow{4}{*}{} & Close proximity to the $\mathrm{Hz}$ & Atmosphere $\sim 1 / 150^{\text {th }}$ that of Earth \\
\cline { 2 - 3 } & Close proximity to Earth $(55-401 \mathrm{x} 106 \mathrm{~km})$ & Wide temperature variations $\left(-125-20{ }^{\circ} \mathrm{C}\right)$ \\
\cline { 2 - 3 } & Light signal takes $3.1-22.4 \mathrm{~min}(\mathrm{av} 12.5 \mathrm{~min})$ to reach Earth & We may disrupt any existing life and natural Martian geological processes \\
\cline { 2 - 3 } & Travel time of $\sim 270 \mathrm{~d}$ & Enormous cost likely detrimental to Earth based projects \\
\cline { 2 - 3 } & Surface gravity $\sim 0.38 \mathrm{~g}$ & \\
\cline { 2 - 3 } & &
\end{tabular}

Opinion: Bronchopulmonary dysplasia (respiratory failure) is the most challenging issue in reducing the minimum gestational age beyond 22 weeks in humans. As technology develops prototype artificial large animal and subsequently human placentas to facilitate optimal liquid to gas exchange required to counteract impaired structural and functional immaturity of the lungs, it may become possible to support extremely premature babies. Today, the concept of programmed and automated ectogenesis is unlikely to have impact on our quest for space colonization though over the next $100 \mathrm{yr}$, it may be possible, subject to appropriate legislation and solving ethical issues to develop a human being to full term outside of a host mother.

\section{Construction and deployment of nano spacecraft}

The most recent example of a nano spacecraft is the StarChip a cm-sized, g-scale interstellar spacecraft proposed for the Breakthrough Starshot program [29] which proposes to launch and propel a fleet of a thousand StarChips on a journey to the Alpha Centauri star system, our nearest star which is approximately 4.37 light year from Earth. It is also proposed that the journey will include a flyby of Proxima Centauri b, a candidate exoplanet within the habitable zone of the host star. A concept previously reported [30], the StarChip is a robotic nano spacecraft, fitted with a lightsail, which is to be propelled by a multi-km phased array of $10 \mathrm{~kW}$ ground-based lasers with a combined output of up to $100 \mathrm{GW}$ 
[31]. The StarChips are planned to travel at speeds of $20 \%$ and $15 \%$ of the speed of light, taking between 20 and 30yr to reach the star system respectively and about 4yr to signal Earth of a successful arrival.

Opinion: There are significant challenges to produce sufficient laser power to propel the StarChips one by one. The light sail is estimated to require an area of between $16-20 \mathrm{~m}^{2}$ and all components must be produced to withstand extreme acceleration $\left(100 \mathrm{kms}^{2}\right.$ experiencing 10,000g), cold, vacuum, protons and collision with space debris [32]. Sending a fleet of approximately 1000 StarChips is a mitigation measure for anticipated losses caused by collision with inter-stellar dust en route to the target. Sprites, precursor prototypes for StarChip, were successfully launched in July 2017 and flown through the Polar Satellite Launch Vehicle by the Indian Space Research Organisation [33]. Development of material sciences supporting the aeronautics business over the next $100 \mathrm{yr}$ is expected to have a positive impact on the probability of technical success. As such, it is anticipated that deployment and successful arrival of nano spacecraft is likely to occur within $100 \mathrm{yr}$ [30] and that this achievement will have major impact on the goals for space colonisation and travel.

\section{Mind-uploading, whole brain emulation (WBE) and avatars}

A controversial and scientifically thought-provoking field is the concept of mind-uploading or whole brain emulation to a hard drive or cloud-based software application. An extreme, if not ultimate form of artificial intelligence, it has preoccupied philosophers particularly since the publication of a roadmap for WBE in 2008 [34]. The concept, in its simplest form is for one or more individuals with pre-defined skills, knowledge and characteristics to have details of their memories, personality and consciousness scanned and captured and that the collective would for an e-crew which has all the benefits of being virtual and none of the hindrances of being in a physical form. Table 3 illustrates some potential opportunities and challenges of generating an avatar via WBE and then deploying it as part of an e-crew on a nano spacecraft. In 2014 [35] the neural network of 302 neurons and the 7,000 neural connections of each neuron of the worm Caenorhabditis elegans (C. elegans) were mapped, a computer code written and the code inserted into a Legobot. The worm's nose neurons were replaced by a sonar sensor on the robot. The motor neurons running down both sides of the worm corresponded to motors on the left and right sides of the robot. Stimulation of the 'nose' stopped forward motion and contact with the anterior and posterior touch sensors made the robot move forward and backward respectively while stimulating the food sensor prompted forward movement $[35,36]$. There are enormous challenges in simulating the neural network of a human brain with its 86 billion neurons and over 100 trillion synapses and as of 2015, the state-of-the-art has been reported [37]. Advances in the fields of medicine such as in vivo brain scanning, deployment of nanodetectors to follow and map neural pathways, the gaming industry and development of further instigation of virtual world existence will rapidly propel this area forward.

Table 3: Generation of an e-crew or avatar-based crew.

\begin{tabular}{|c|c|}
\hline Opportunities & Challenges \\
\hline No limitation of human lifespan & $\begin{array}{l}\begin{array}{l}\text { Viability depends on component lifetime needing to last hundred or thou- } \\
\text { sands of yrs }\end{array}\end{array}$ \\
\hline No limitation for travel time & $\begin{array}{l}\text { An avatar without physical robotic form will be severely restricted in build- } \\
\text { ing a colony }\end{array}$ \\
\hline $\begin{array}{l}\text { No need for air, water, food, medical care, radiation shielding, protec- } \\
\text { tion against extreme acceleration. }\end{array}$ & \multirow{3}{*}{$\begin{array}{c}\text { Legal and ethical challenges } \\
\bullet \text { Who to select? } \\
\text { •Is an uploaded consciousness human? } \\
\text { •How to monitor and terminate activities if needed? } \\
\text { •How to prevent conflict between avatar and self? } \\
\text { •How to co-ordinate activities of an e-crew? }\end{array}$} \\
\hline More compatible with the size and weight of a nano spacecraft & \\
\hline $\begin{array}{l}\text { Advanced forms of AI fulfill mission requirements while not risking } \\
\text { human life in the process }\end{array}$ & \\
\hline
\end{tabular}

Opinion: Long term plans to develop a synthetic C. elegans hologram to host the digital connectome of the worm are progressing [36], assisted by physical simulations of soft tissue [38]. Mapping human neural networks has utility for increased understanding of neurological, neurobehavioral and neuropsychiatric disorders and may provide more knowledge on how we think, reason and negotiate, particular in a closed or confined environment such as a digital e-crew on a long-term space mission. It appears likely that within the next $100 \mathrm{yr}$, construction and development of an independent and self-contained android existence will have been achieved [37], which will have significant impact and coupled together with the development of nano spacecraft, it may be within the realms of possibility that space travel and survey exoplanets surrounding distant stars is attainable over this time period. This may be expected to have a major impact on our goal for deep space travel and colonization, although settlement as such may be potentially as virtual human beings.

\section{Other concepts revisited}

Previously I discussed the possibilities of interstellar travel via technologies proposing to travel faster than light (FTL), the potential for human life extension via stasis or torpor, engineered negligible senescence or cryogenics and the concept of multigenerational voyages via Worldships [15].

Opinion: There are two areas for which scientific development may render an increased likely hood of success and therefore greater impact on our goal for space colonization. Placing astronauts into a state of torpor by therapeutic hypothermia to support prolonged hypothermic stasis and metabolic suppression 
while providing total parenteral nutrition is the first step. This needs to be accompanied by prevention of muscle atrophy, for example, by electrical stimulation and the crew experiencing artificial gravity to ameliorate the effects of microgravity and is part of the aim at the Space Works organization [39]. It is proposed that to experience $1 \mathrm{~g}$, that is Earth's gravity a habitat of $5.5 \mathrm{~m}$, a size deemed commercially viable needs to rotate at $18 \mathrm{rpm}$. Such a rotation speed would be unacceptable if the astronaut was fully conscious but may be permissive if the astronaut was in stasis. It is likely that such a hibernative state will be explored for astronaut travel to near term planets and that Mars is the natural candidate within the next 100yr. Research will continue in the science of ageing and the biology will progress, based in part on in depth analyses of the physiology and biochemistry of some members of the animal kingdom whose life span exceeds $200 \mathrm{yr}$. It is deemed possible that over the next $100 \mathrm{yr}$, extending human lifespan by engineering negligible senescence may have a part to play in long term space missions.

\section{Conclusion}

Travel to and colonization of bodies within our solar system is making progress with colonization of Mars expected to occur through both public and privately funded consortia in the 2020s and 2030s [40-42]. This will be a significant step forward to preparing for future deep space travel and may mark a decision point as to whether to continue with human bodied space travel or to divert part or all our resources to some form of AI or e-crew capability. In the event of the latter and assuming that at least $30 \mathrm{yr}$ will elapse before data is received and analyzed from our nearest candidate exoplanet, it will be essential to retain public and scientific interest and support in the future of space travel and not to dismiss the possibility of human bodied space travel and colonization, possibly of terraformed bodies in the solar system. Maintaining a balanced view in the media will be also essential and as expectations are met and the possibility of climate change becomes reality, this may need to become more objective. Our species has challenging times ahead and the biggest challenge to date is to ensure that planet Earth remains a viable, self-sustained world, that Holocene period is short and that together, we harness our tremendous global resources and ingenuity to preserve our species and to continue to explore, travel and ultimately colonise the universe in which we live.

\section{Acknowledgement}

Martin Braddock is a member of the Mansfield and Sutton Astronomical Society (MSAS) a Fellow of the Royal Society of Biology and a Fellow of the Royal Astronomical Society. MSAS is a registered charity, number 51813 and was founded in 1970 to foster interest in astronomy in Ashfield, Nottinghamshire, United Kingdom and surrounding district.

\section{References}

1. http://phl.upr.edu/projects/habitable-exoplanets-catalog

2. https://exoplanetarchive.ipac.caltech.edu/

3. Perryman M, Hartman J, Bakos GA, Lindegren L (2014) Astrometric exoplanet detection with Gaia. Astrophys J 797: 1-22.

4. https://www.cosmos.esa.int/web/gaia

5. Livingston JH, Endl M, Dai F, Cochran WD, Barragan O, et al (2018) 44 validated planets from K2 campaign 10. Earth and Planetary Astrophysics, pp. 1-25.

6. https://www.nasa.gov/mission_pages/kepler/main/index.html

7. https://tess.gsfc.nasa.gov/

8. https://tess.gsfc.nasa.gov/documents/TESS_FactSheet_Oct2014.pdf

9. Pimm SL, Jenkins CN, Abell R, Brooks TM, Gittleman JL, et al (2014) The biodiversity of species and their rates of extinction, distribution and protection. Science 344(6187): 1246752.

10. Ceballos G, Ehrlich PR, Barnosky AD, Andrés G, Robert MP, et al. (2015) Accelerated modern human-induced species losses: entering the sixth mass extinction. Science Advances 1(5): e1400253.

11. Braddock M (2017) Ergonomic challenges for astronauts during space travel and the need for space medicine. J Ergonomics 7: 1-10.

12. Shiba D, Mizuno H, Yumoto A (2017) Development of new experimental platform 'MARS'-multiple artificial-gravity research system-to elucidate the impacts of micro/partial gravity on mice. Scientific Reports Vol. 7.

13. Clement G (2017) International roadmap for artificial gravity research. Npj Microgravity 3: 29.

14. Braddock M (2017) Artificial gravity: small steps on the journey to the giant leap. J Space Explor 6: 137-145.

15. Braddock M (2018) Concepts for deep space travel: from warp drives and hibernation to world ships and cryogenics. Curr Trends Biomed Eng Biosci 12.

16. Albert S, Leon JX, Grinham AR, John AC, Badin RG, et al. (2016) Interactions between sea-level rise and wave exposure on reef island dynamics in the Solomon Islands. Environ Res Lett Vol. 11.

17.https://ec.europa.eu/clima/policies/international_en

18. http://www.wri.org/sites/default/files/wri14_factsheet_climateaction. pdf

19. http://www.un.org/en/sections/issues-depth/climate-change/

20. Raup DM (1985) Magnetic reversals and mass extinctions. Nature 314: 341-343.

21. Long J, Large RR, Lee MST, Michael JB, Leonid VD, et al. (2015) Severe selenium depletion in the Phanerzoic oceans as a factor in three global mass extinction events. Gondwana Res 36: 209-218.

22.http://www.nasa.gov/press-release/goddard/2018/marsterraforming

23. Jakowsky BM, Edwards CS (2018) Inventory of $\mathrm{CO}_{2}$ available for terraforming Mars. Nature Astron 2(8): 634.

24. Way MJ, Del Genio AD, Kiang NY, Linda ES, David HG, et al. (2016) Was Venus the first habitable world of our solar system? Geophys Res Lett 43: 8376-8383.

25. Adamson GD, Tabangin M, Macaluso M, Mouzon J (2013) The number of babies born globally after treatment with the assisted reproductive technologies (ART). Fertility and Sterility 100(3): S42.

26. https://www.simplemost.com/at-least-8-million-ivf-babies-have-beenborn-in-the-last-40-years/

27. Bulletti C, Palagiano A, Pace C (2011) The artificial womb. Ann NY Acad Sci 1221: 124-128.

28. Partridge EA, Davey MG, Hornick MA, McGovern PE, Mejaddam AY, et al. (2017) An extra-uterine system to physiologically support the extreme premature lamb. Nature Communications 8: 15112. 
29. http://www.centari-dreams.org?p=3502.

30. Lubin P (2016) A roadmap to interstellar flight. J Brit Interplan Soc 69: 40-72.

31. http://www.breathroughinitiatives.org/Concept/3.

32. h t t p : / / b r e a throughinitiative s.org/index. php?controller=Forum\&action=viewforum\&id=6\&page $=1$.

33. http://breakthroughinitiatives.org/News/12.

34. Sandberg A, Bostrom N (2008) Whole brain emulation: A roadmap, technical report \#2008-3, Future of Humanity Institute, Oxford University, USA.

35. https://www.smithsonianmag.com/smart-news/weve-put-wormsmind-lego-robot-body-180953399/?no-ist
36. http://openworm.org/

37. Feierbach G, Kroene RA (2016) Whole brain emulation-state of the art.

38. Palyanov A, Khayrulin S, Larson SD (2016) Application of smoother particle hydrodynamics to modeling mechanisms of biological tissue. Adv Eng Software 98: 1-11.

39. http://www.sei.aero/archive/SpaceWorks_NIAC_Symposium_2016_ Final.pdf

40. http://www.artificial-gravity.com/sw/SpinCalc/SpinCalc.htm

41. http://www.mars-one.com/mission/roadmap

42. http://www.planetary.org/blogs/jason-davis/2017/20170929-spacexupdated-colonization-plans.html. (c) (i) Creative Commons Attribution 4.0 International License

For possible submissions Click Here

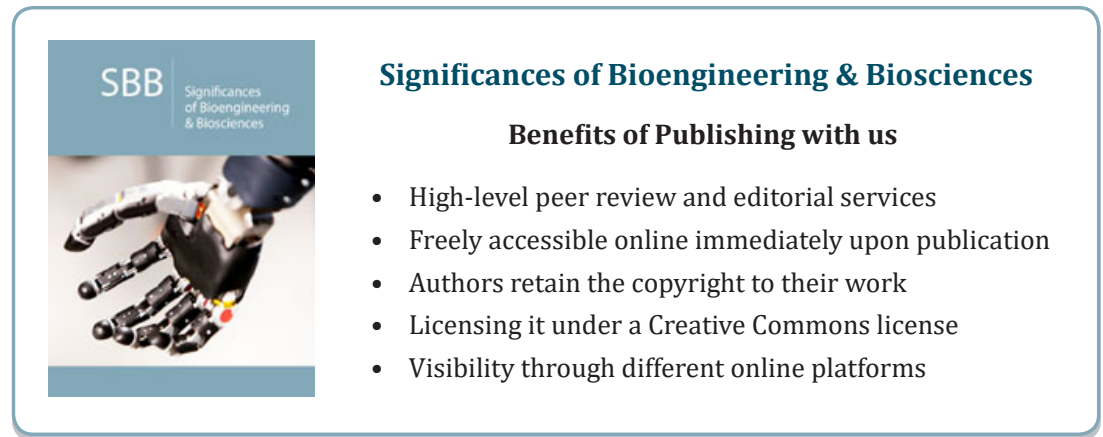

